\title{
EL PATRIMONIO ERA OTRA COSA
}

\author{
Felipe Criado-Boado y David Barreiro
}

"Si la mirada es una pérdida, hay que saber conservar al menos la pérdida misma. No podemos vivir -ni cuidar nada- sin nostalgias y sin enigmas". Santiago Alba Rico

\section{* Introducción}

\section{Resumen}

En el presente texto se destacan algunos rasgos sobre el valor actual del patrimonio cultural, la situación de los estudios de investigación y gestión de dicho patrimonio, la necesidad de conjugar múltiples perspectivas y narrativas en la interpretación de éste, la conveniencia de establecer un sistema ordenado de diálogo entre todos los intereses

y perspectivas que en él confluyen, la interacción de los proyectos patrimoniales con las comunidades y, en definitiva, la construcción de un patrimonio público. ${ }^{2}$

Palabras claves: patrimonio cultural - patrimonio público - cultura material - estudios culturales - arqueología pública.

This text deals with the present value of cultural heritage and successively considers: the current situation of studies about research

and management of this heritage, the needs to combine multiperspective and multi-narrative approaches for its interpretation, the advantage for establishing an arrangement to promote dialog

between different interests and perspectives, the interaction of heritage projects with local communities and, therefore, the project to build up a public heritage.

Key words: cultural heritage - public heritage - material culture cultural studies - public archaeology.

Recibido: noviembre 2012. Aceptado: febrero 2013.
En este trabajo vamos a plantear algunas consideraciones sobre la concepción y pragmática del patrimonio cultural (al que nos referiremos, en lo sucesivo y casi siempre, como "patrimonio") agrupando cuatro tipos de argumentos principales. Primero, sobre el concepto y definición de patrimonio. Después, examinando las cualidades que se sedimentan sobre el patrimonio. A continuación, revisando las consecuencias de todo ello para la pragmática del patrimonio. Y finalmente, identificando algunas prioridades de estudio y trabajo en relación con los bienes patrimoniales.

\section{* Concepto}

Arqueología, antropología, etnografía, etnología, historia, historia del arte, arquitectura, estudios culturales, semiótica, historia de la ciencia, restauración... todas estas prácticas tienen que reformularse para abordar el patrimonio como objeto de trabajo y para adaptarse a las necesidades teórico-prácticas que éste plantea. La noción de patrimonio es central a todas ellas, aun cuando su vertiente más académica (en cierto modo, la más distante de la realidad del patrimonio) no lo tenga en cuenta, o incluso, rechace explícitamente esta idea. Podríamos ir más allá: la noción de patrimonio es hoy central a la experiencia y circunstancias de nuestras sociedades mul-

1 Instituto de Ciencias del Patrimonio-Consejo Superior de Investigaciones Científicas (Incipit-CSIC). Equipo del Programa de Investigación para la Conservación y Revalorización del Patrimonio Cultural, también conocido como CSD-TCP. San Roque 2, 15704, Santiago de Compostela, ESPAÑA. Email: felipe.criado-boado@incipit.csic.es; david.barreiro@incipit.csic.es

2 Este texto parte de las ideas defendidas por el primer autor en el VII Congreso Nacional de Prospectiva del Perú, organizado por Fernando Ortega, del CONCYTEC, y celebrado en Cusco entre el 10 y 11 de septiembre de 2009, complementadas con las ideas sustentadas por el segundo autor. 
ticulturales y a la producción de sentido dentro de ellas. Uno se puede preguntar por qué. Pero, ante todo, hay que intentar explicar(se): ¿qué es, entonces, el patrimonio cultural?

Diciéndolo de forma breve, el patrimonio se puede comprender como la huella de la memoria y el olvido. De forma más larga, sirviéndonos de la definición que utilizamos en el Plan Estratégico de nuestra Unidad de Investigación (el Instituto de Ciencias del Patrimonio, del CSIC, en Santiago de Compostela, España), esa huella la compondrían: el conjunto de productos de la actividad humana y sus restos, intencionales y no intencionales, tangibles ( 0 materiales) e intangibles (o imaginarios), que representan los procesos históricos y sociales, que obtienen su sentido de éstos y que, al tiempo, son resignificados por ellos al introducir sentidos nuevos y/o ser renegociados (esto es, los propios procesos de patrimonialización; Smith 2006 , 2011; Sánchez-Carretero 2012). Esta definición incluye, en primer lugar, a los entes materiales e ideas que son socialmente valorados como "bienes patrimoniales" (cuando nos refiramos al patrimonio como objeto de trabajo, lo haremos en un sentido epistemológico, es decir, aquello que nos es dado conocer, independientemente de su corporeidad o incorporeidad y de su condición de producto o proceso), ya sea en el propio contexto en que son creados o en otro contexto posterior. En segundo lugar, incluye a los "entes materiales" y "restos" factibles de alcanzar esa valoración más tarde por su carácter representativo (objetos susceptibles de ser considerados patrimoniales; lo que González-Pérez y Parcero-Oubiña [2011] denominan "entidad primaria"). En tercer lugar, esta definición entiende que el patrimonio es un hecho metacultural, es decir, un hecho social mediante el cual ciertos procesos de sociedades distintas y momentos anteriores, y/o sus impresiones materiales (sus productos, intencionados o no) se legitiman como algo digno de ser estudiado, conservado, inventariado, catalogado y puesto en valor: es decir, son socialmente apreciados (y utilizamos aquí el adverbio "socialmente" conscientes de la ambigüedad y vaguedad que implica a priori, su empleo; pero trataremos de arrojar alguna luz sobre esto).

Por ello, la definición incorpora, además, lo que de forma errónea se denomina "patrimonio natural", no solo porque el medioambiente es, siempre, un producto influido por la acción social y que guarda la huella ecológica de la humanidad, sino también porque el medio natural alcanza la categorización de "patrimonio" a partir de una semantización sociocultural (Criado-Boado et al. 2008). En este sentido es interesante el debate sobre la idoneidad, temporalidad y características del concepto "antropoceno", bien sintetizado en el epígrafe inicial de Solli y colaboradores (2011) y en Chesworth (2012), y al que el Incipit, entre otros, ha contribuido en ocasiones (Kaal et al. 2011), señalando la incidencia ambiental de la actividad humana en épocas muy anteriores a la era industrial (que es a la que algunos autores vinculan el término "antropoceno").

En suma, este planteamiento quiere decir que el patrimonio existe ahora, pero no ha existido siempre; antes había otras cosas que no eran todavía patrimonio. Como tal, el patrimonio solo existe ahora y en el ahora. Y es que el patrimonio, igual que las ciencias humanas y sociales que lo significan (aunque no únicamente lo hagan ellas), es un fenómeno moderno (Hernández 2008). Para que se dé un proceso de patrimonialización (de aprecio social hacia una entidad primaria dada que, así, deviene en objeto patrimonial) tiene que darse una serie de fenómenos culturales (memoria colectiva, vínculos identitarios, creación de un sentido del lugar) que no son específicamente modernos. Pero sí que es moderno el modo en que dichos fenómenos se formalizan a nivel del discurso y de la práctica. En primer lugar, porque la patrimonialización se asocia a un deseo de conservar lo dado que obedece a una conciencia del cambio social y de la pérdida colectiva, que es típicamente moderna (Berman 1988). En segundo lugar, porque es en la modernidad donde los procesos de apropiación del pasado asumido como propio, o de la otredad, que no son exclusivamente modernos, se cristalizan en una visión presuntamente objetiva de ese pasado y de esa otredad, dando paso a procesos de reificación propiamente modernos, anclados en la dualidad sujeto-objeto, lo que no tiene por qué impedir que esos otros procesos de apropiación premodernos (más bien habría que pensar en utilizar conceptos alternativos, como "a-modernos") no se mantengan más o menos ocultos bajo (o marginados por) el discurso hegemónico de la modernidad y la modernización. En este sentido, el concepto de patrimonio tal y como lo entendemos, para bien y para mal, es un concepto alumbrado por la racionalidad moderna. ${ }^{3}$

\footnotetext{
3 Barreiro (2012) lo ejemplifica siguiendo un ejercicio tomado de Harvey (2001).
} 


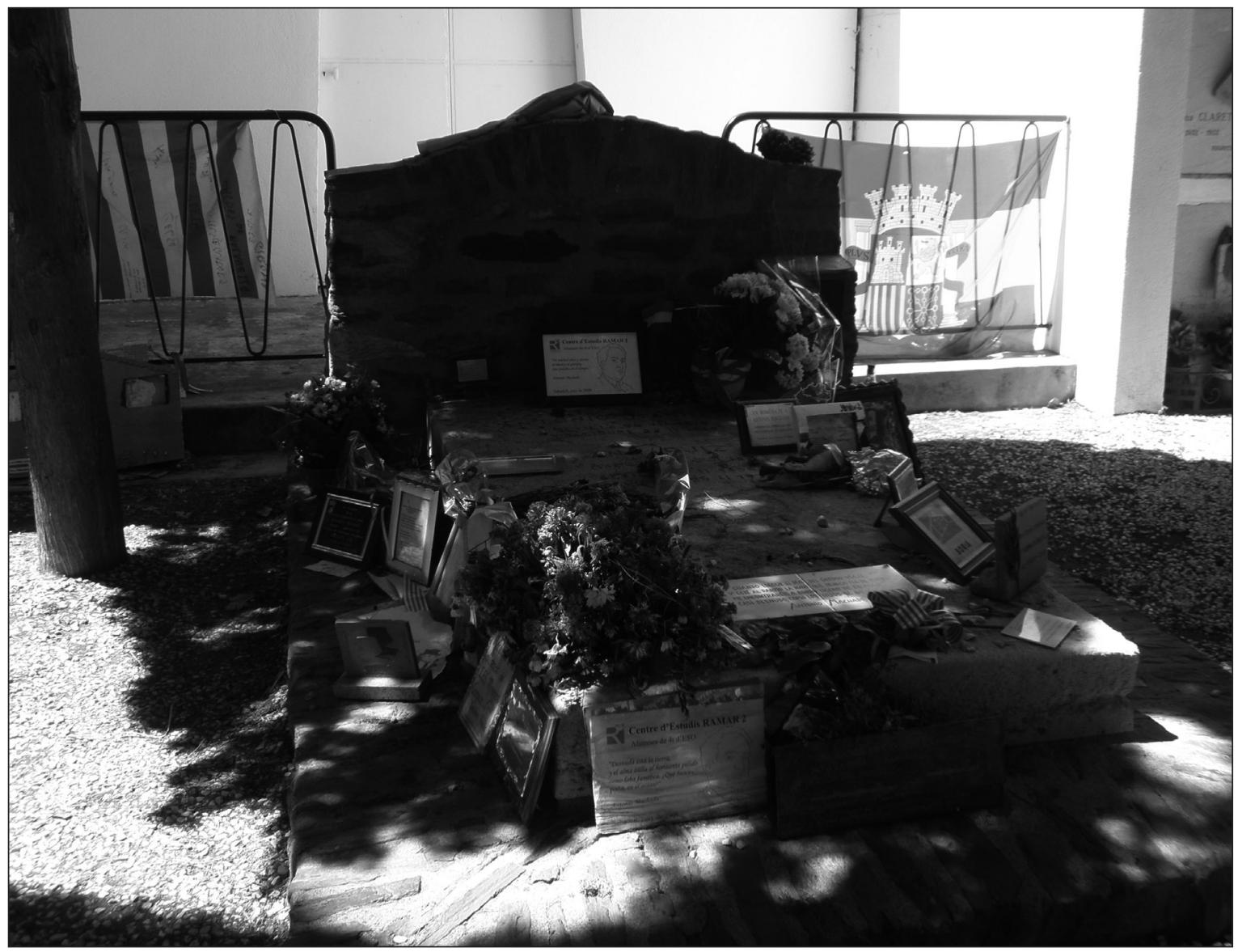

Figura 1. Tumba de Machado.

Todo esto tiene implicaciones importantes para el patrimonio, pues justifica y establece como inevitable la ruptura presente-pasado, la escisión entre el origen y la actualidad del patrimonio, la división entre el contexto de producción de lo que va a ser patrimonio y el contexto de aprecio y valorización que convierte eso en patrimonio. Se podría decir que aquí radica el "pecado original" del patrimonio (si bien esta expresión induce una connotación de culpabilidad e intencionalidad que no se le puede demandar al pasado en nombre del presente), ya que esa separación es lo que determina que el patrimonio sea en gran medida un significante vacío que puede ser rellenado con cualquier significado y, por lo tanto, manipulado para adaptarlo a cosas que tienen menos que ver con su contexto de formación que con el contexto en el que se utilizan y revaloran. Al fin y al cabo, en esto consiste la reificación (Figura 1).

\section{$*$ CAPAS}

Esta realidad ambivalente (antigua y actual, pretérita y presente, nuestra y de otros) del patrimonio multiplica los estratos y complejidades de uso y valoración sobre él. Revisaremos algunos de ellos haciendo una glosa de ciertos enunciados principales que a continuación detallamos.

\section{La realidad del patrimonio es su gestión}

Esto permite mitigar en parte su complejidad, pues de hecho es una calidad transitiva, ya que es tanto consecuencia de la misma como una forma de solucionarla. En efecto, no nos queda más remedio que llegar a la gestión, al manejo reflexivo del patrimonio: si los bienes patrimoniales no existieron en el pasado, sino que son creados por actos de aprecio hechos desde contextos actuales que reutilizan 


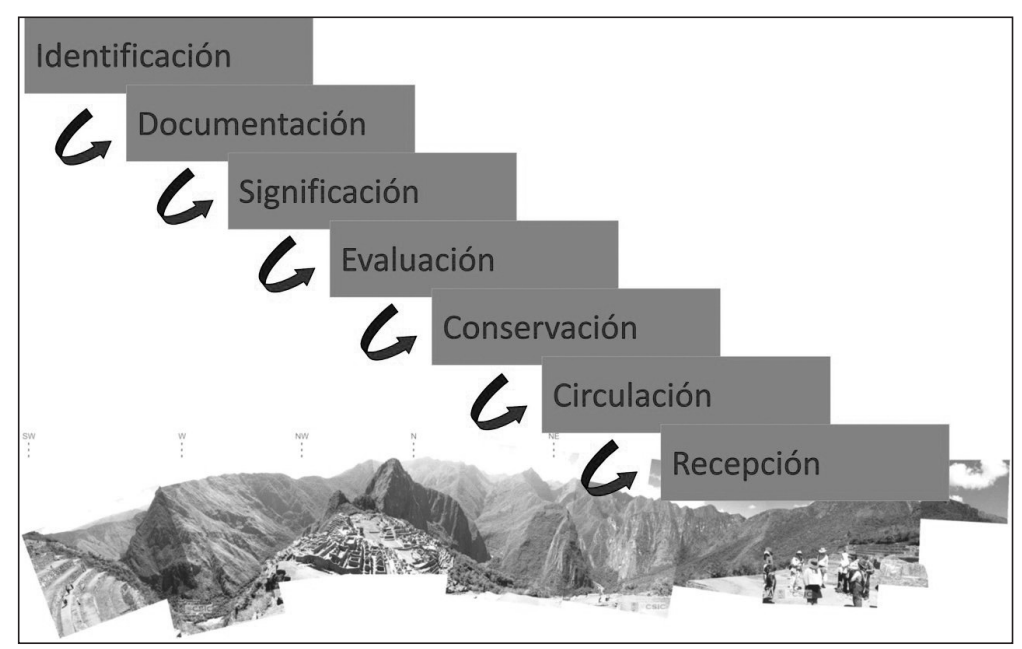

Figura 2. La cadena de valor del patrimonio. Secuencia de fases valorativas que amplían hermenéuticamente el proceso de reconocimiento y socialización de los bienes patrimoniales.

elementos del pasado (o de otros contextos culturales), y si la distancia entre forma patrimonial y sentido original facilita la manipulación de esos bienes (su manejo como free-meaning values), entonces nuestra obligación es propiciar un sistema de gestión integral de esos bienes en el presente que tome en cuenta todas sus dimensiones para gestionarlos debidamente, de forma ordenada $y$, sobre todo, atendiendo a la multiplicidad de valores y prácticas sociales y discursivas en las que se insertan.

\section{La gestión del patrimonio debe ser integral}

Esto implica utilizar la cadena de valor del patrimonio como modelo teórico para organizar la investigación y gestión de los bienes patrimoniales. Este modelo, a cuyo desarrollo ha contribuido de forma significativa la investigación de nuestro laboratorio (Criado-Boado 1996; González-Méndez 1999; Barreiro 2003; SánchezCarretero 2012), entiende que las entidades primarias se transforman en bienes patrimoniales por actos de identificación, documentación, significación, valoración, difusión, circulación y recepción, y establece que las buenas prácticas en investigación y gestión de esos bienes deben incluir todas esas dimensiones, haciéndolo además, de forma:

- sistemática: sin saltarse pasos, pues a menudo la manipulación de los bienes patrimoniales deriva precisamente de esto, es decir, del hecho de no tener en cuenta determinados valores asociados a esos bienes;

- completa: integrando las distintas dimensiones de la práctica científica, desde la generación de conocimiento hasta su aplicación y transmisión;

- reflexiva: incorporando criterios de autoevaluación;

- transdisciplinar: en tanto el propio proceso de valorización del patrimonio es susceptible de convertirse en objeto de estudio para disciplinas no específicamente patrimoniales, como la sociología, la economía, las ciencias de la información y de la educación, entre otras;

- participativa: abriendo el proceso e integrando la valoración no experta (Figura 2).

\section{La controversia es inherente al patrimonio}

Los entrecruzamientos de orígenes, razones, intereses, actores, sentidos y legitimidades, hace que el patrimonio sea algo siempre disputado y en discusión; los bienes patrimoniales son el escenario preferente de negociaciones y conflictos; los motivan cuando diferentes intereses se justifican sobre su patrimonio, y los sufren cuando actores en pugna toman como rehén el patrimonio de los otros. Desde la quema de la Biblioteca de Alejandría has- 
ta la de Sarajevo y la destrucción de patrimonio durante las últimas guerras de los Balcanes, y todas las guerras, (recordemos el caso de los Budas de Bamiyán), son buen ejemplo de ello. Esta idea nos acerca a la de aquellos especialistas que entienden el patrimonio más como proceso abierto y negociado que como objeto o cosa (Waterton y Smith 2009: 10-28) ${ }^{4}$, aunque no nos sitúa en su misma perspectiva, y nos aleja (aunque no nos divorcia) de la de aquellos especialistas que lo entienden como objeto o producto cerrado en sí mismo, instrumentalizable e intrínsecamente valioso. Y es que la ambivalencia del concepto patrimonio se manifiesta igualmente cuando intentamos discernir su real naturaleza, a la vez acción y objeto, producción y producto, verbo y sustantivo. Este nadar entre dos aguas sin hacer pie es nuestro ámbito de trabajo: el patrimonio es un concepto dialéctico; es, al mismo tiempo que era y será otra cosa; de hecho, es una cosa, pero una cosa en continua transformación, y dentro de esa cosa estamos todos, objetos y sujetos, entes y agentes de patrimonio en un movimiento perpetuo.

\section{Existe un patrimonio indeseado 5}

El patrimonio no es solo el monumento de las memorias victoriosas en la historia. Los perdedores, los protagonistas anónimos, las resistencias, también producen bienes patrimoniales. La voluntad de verdad y la voluntad de visibilidad hegemónicas pueden ocultar esto y condenar otros patrimonios al olvido y la opacidad. Pero los materiales son tan persistentes como las resistencias, y a través de aquellos finalmente se representan éstas. Así terminan emergiendo patrimonios no deseados por el poder dominante, pero que se hacen presentes y reafirman la presencia y reclamaciones de otras voluntades. La Cárcel de Carabanchel en Madrid, sería un ejemplo. Fue la principal prisión de la represión franquista contra los opositores al régimen y recientemente fue demolida dentro de una operación inmobiliaria, pero, mientras tanto, la comunidad y los memoriosos de la resistencia demandaban su preservación como monumento de memoria. Así, se convirtió en ejemplo de un patrimonio no deseado (González-Ruibal 2009). La cuestión clave que hay que responder es: ino deseado por quién? En realidad, si el patrimonio es un proceso

\footnotetext{
4 Ver también Smith (2006) y Santamarina (2005).

5 Tunbridge y Ashworth (1996).
}

controvertido, conflictivo en sí mismo, en todo proceso de patrimonialización van a existir sujetos "no deseantes" (no es el patrimonio lo que no es deseado; sino que son los sujetos que hacemos el patrimonio, expertos y no expertos, los que podemos no desearlo). El patrimonio, por sí mismo, al igual que la modernidad, no es algo positivo ni negativo; es un medio para la acción social y cultural, un campo en el que es factible poner en juego discursos enfrentados y luchar por la emergencia de formas de sociabilidad alternativas, basadas más en relaciones solidarias que en intereses individuales. Es esta una visión dialéctica, que asume que cualquier trascendencia de la modernidad (por eso hablamos de transmodernidad) tiene que producirse dentro de su práctica discursiva y que el patrimonio es un terreno más en el que esas contradicciones en el seno de la racionalidad moderna hegemónica se manifiestan, como formas contrahegemónicas, más o menos incipientes, emergidas en el seno de su propia práctica discursiva - como el pensamiento emancipador y utópico- o directamente como racionalidades no modernas que han sido subsumidas - pero desigualmente integradas en el discurso de la modernización, como las múltiples racionalidades indígenas que pugnan cada vez más por manifestar su propia voz, su propia memoria y su propia identidad dentro de la modernidad, reclamando su propio modo de modernización. Creemos que, salvando las diferencias entre los variados contextos geopolíticos, esto último es lo que se está produciendo hoy en día en casi toda Latinoamérica, concretado en múltiples ejemplos a nivel micro (p.e., el caso de la exposición de las momias de antiguos atacameños en el Museo de San Pedro de Atacama y su posterior retirada por la presión de las comunidades), y macro (p.e., los conflictos en Ecuador y Bolivia en torno al impacto de proyectos industriales y desarrollistas sobre los recursos patrimoniales, naturales y culturales, y su incardinamiento en una práctica discursiva modernizadora que pretende, simultáneamente, absorber dentro de sí el alter discurso indigenista).

\section{El patrimonio es el lugar de cruce de diferentes racionalidades culturales}

A menudo esas intersecciones propician, como consecuencia de las dos cualidades anteriores, un choque de lógicas del mundo. Esta cualidad del patrimonio es inherente a él 


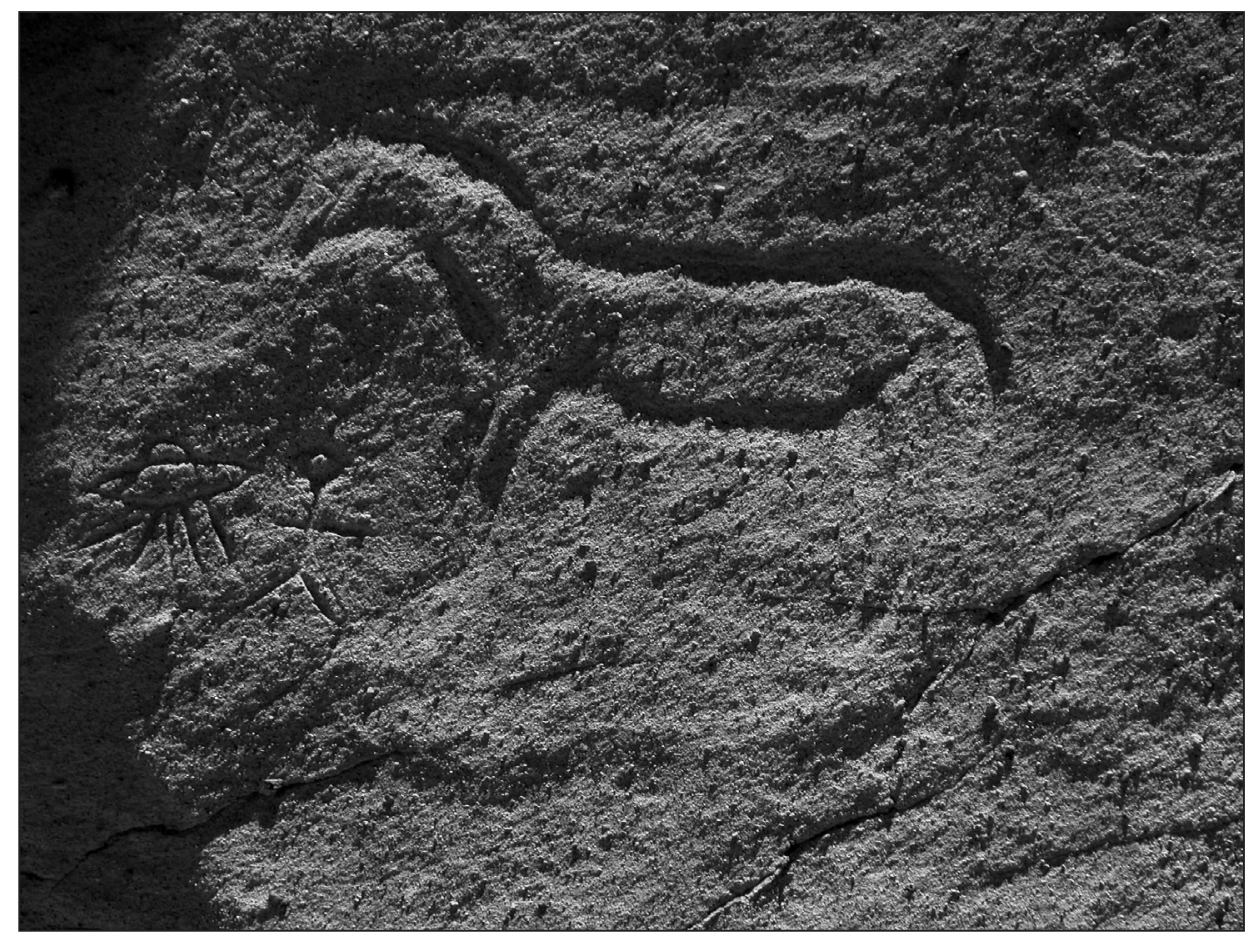

Figura 3. Extraterrestres en Yerbabuena, cerca de San Pedro de Atacama. En este cruce de caminos del desierto, se localiza un importante sitio de arte rupestre conformado por la superposición de representaciones de diferentes épocas. Entre ellas aparece un ejemplo supino de vandalismo patrimonial: un bromista extraviado, un desalmado malintencionado o un new age desorientado ha grabado un OVNI dentro del conjunto. Pero en la misma medida en que es una agresión al patrimonio, es un acto que lo resignifica y que introduce otra capa de sentido en el conjunto arqueológico.

porque no en vano los bienes patrimoniales suponen el lugar de encuentro de, cuando menos, dos racionalidades: la original y la actual, con sus respectivos pliegues, a los que antes aludíamos. En realidad son muchas más las racionalidades que en ellos confluyen, pues, entre ambos extremos (el original y el actual), el patrimonio ha sido valorado y utilizado por múltiples contextos de recepción y uso, cada uno de los cuales converge en él con su propio discurso y racionalidad. Es más, dado que los actos de patrimonialización toman ventaja de la tradición y prestigio previo de algunos bienes patrimoniales, las superposiciones son frecuentes. Pero toda superposición de acontecimientos es una superposición de racionalidades. La Mezquita de Córdoba desde los omeyas hasta los cristianos es un buen ejemplo. Como también lo es el Koricancha en Cusco, la yuxtaposición de grabados de diferentes épocas (desde el Formativo a la Colonia) en diferentes sitios de arte rupestre del Norte Grande, o las propias momias del Museo de San Pedro de Atacama. En todos estos casos, es la su- perposición la que confiere a los bienes toda su complejidad, pero es la acción social presente la que le otorga valor patrimonial, y esta acción presente puede ser más viva, multivocal y participativa, pero también puede obedecer a una lógica "cosificadora" que acaba por dotar de valor solamente a la encarnación material de esos espacios (a los edificios, a los grabados) y, en todo caso, también a sus réditos económicos (cuando los hay), en lugar de a su condición de bienes patrimoniales en toda su complejidad y multidimensionalidad (Figura 3).

\section{El patrimonio es el acervo de las sociedades}

Lo que entendemos como "patrimonio" (ya sea desde una posición "occidental" o desde la posición de los pueblos originarios que, como parte de su proceso de modernización, asumen el discurso patrimonial), constituye en realidad el repositorio que acumula las tradiciones culturales de diferentes comunidades y grupos. 


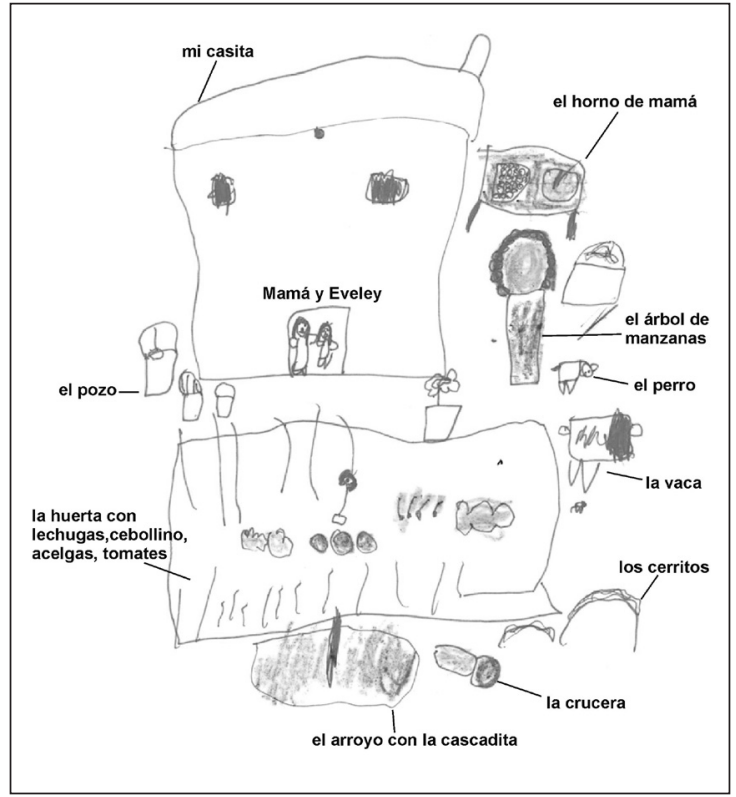

Figura 4. El mundo de Eveley. Dibujo de una niña de cinco años de la escuela de Pago Lindo (Caraguatá-Tacuarembó) en Uruguay. El dibujo es un auténtico mapa cognitivo de la realidad de Eveley. Vive sola con su madre, en un medio rural aislado y empobrecido. Desde la puerta de su choza, centro de su mundo, en cuyo umbral aparecen Eveley y su mamá, se despliega todo lo que es importante para ella. Las actividades de concienciación

patrimonial y participación comunitaria en el trabajo arqueológico que se realizaron en el proyecto de investigación allí realizado por el Incipit y el Laboratorio de Arqueología del Paisaje y Patrimonio de Uruguay (LAPPU) -tales como charlas, talleres, visitas a los sitios arqueológicos, cortos recorridos de prospección por el terreno circundante- le permitió tomar conciencia de los cerros que se encuentran a escasos $100 \mathrm{~m}$ de su casa, marcando

de hecho el límite entre su ámbito doméstico y el resto del mundo. Es un buen ejemplo de cómo el patrimonio arqueológico sirve para crear el sentido del lugar y reforzar los lazos incluso individuales con el entorno. La percepción de ellos por parte de Eveley le confiere una conciencia ampliada de su realidad. Tomado de Gianotti y colaboradores (2011).

Sus materiales, tecnologías, formas de enfrentarse al mundo, conocimientos, valores, símbolos y aparato sociocultural, se representan y contienen en el patrimonio. El patrimonio fosiliza, pero no siempre en la misma medida. El grado de fosilización no tiene por qué implicar que el patrimonio exista por sí mismo, como una entidad esencial, sino que depende enteramente de los agentes de patrimonialización y de los valores que entran en juego en aquel proceso.

\section{El patrimonio no existe en sí o para sí, sino de sí}

La razón de ser del patrimonio no se agota en sí misma, del mismo modo que el patrimonio no se constituye por sí mismo. Depende de instancias externas a él (los agentes de patrimonialización) que lo convierten en patrimonio al significarlo y valorizarlo (en todos los sentidos correlacionados del término). Por eso no es un ser-en-sí ni un ser-para-sí, sino que es un ser-de-sí para el mundo. Esto se relaciona con lo siguiente.

\section{El patrimonio se debe al aprecio de los ciudadanos}

Es el resultado de actos de valoración y estima que permiten reconocer al patrimonio como algo propio o reconocible por ciertos sectores, cuando no por la totalidad de una sociedad. Pero eso quiere específicamente decir que el patrimonio no se puede separar de la estima que los pueblos, comunidades e individuos sienten por él, y por lo tanto su protección, gestión y conservación no se resolverá debidamente sin tener en cuenta esta valoración. Sin un acto inicial de aprecio el patrimonio no llega a ser tal; pero tampoco se puede conservar y mantener sin el aprecio posterior. Lo que no se aprecia, deja de ser valorado como patrimonio y su conservación plantea entonces problemas prácticos que están a la altura del problema epistémico y ético, incluso lógico, que es poder reconocer algo que no se quiere conocer. La conservación del patrimonio arqueológico en contextos conflictivos es un buen ejemplo. Por motivos identitarios, culturales o económicos, el patrimonio suele contravenirse con nuevos usos urbanos o intereses mineros, al contraponerse a proyectos de producción de valor que interesan a determinados agentes $y$, a veces, a la mayor parte de la comunidad. Un mosaico romano en una ciudad es patrimonio que se debe conservar, pero si no hay una corriente de reapreciación, su conservación plantea problemas esenciales y a menudo irresolubles; no hay alternativa frente a la presión especulativa, salvo la conservación a ultranza, que no es una buena aliada para resolver los problemas de forma pragmática (Figura 4).

\section{La socialización del patrimonio es precondición para su preservación y conservación}

Esto tiene una implicación práctica, pues las estrategias y proyectos de revalorización (concepto que implica tanto 
resignificar como fomentar la estima o acometer acciones efectivas de puesta en valor, a pesar de que a menudo se olvida y se confunde con una intervención física o constructiva para poner en valor) no son algo accesorio ni algo anexo a algunos proyectos privilegiados, sino un planteamiento que tiene que estar en la raíz de las estrategias de conservación, que tiene que surgir en el mismo momento que se generan las alternativas sobre el qué hacer con los bienes patrimoniales.

Esto nos retrotrae al argumento anterior del patrimonio no deseado y el agente no deseante. Cuando hay una mayoría de la población que no desea la conservación del patrimonio, ¿qué criterio puede legitimar una postura opuesta? Habría una solución fácil pero compleja, que raya casi en la metafísica: aceptar que el derecho esencial de todo lo que existe (sea criatura viva o no) es seguir existiendo. Esto, además, plantea problemas prácticos: ¿cómo conciliar el derecho del todo a seguir existiendo? Aunque aquí emerge una bonita noción de desarrollo sostenible, que diría que es sostenible aquel desarrollo que concilia los derechos de todo lo existente, mientras no desarrollemos más una conciencia y una práctica global de esta forma de sostenibilidad, tememos que para resolver el dilema de la conservación del patrimonio, no nos queda más remedio que acudir a instancias de legitimación externas (p.e., la autoridad intelectual). Todo esto se relaciona con el problema de la democratización de la gestión del patrimonio, en la que la respuesta está en él mismo: no es que se deba o pueda educar a la gente para que desee conservar su patrimonio, sino que en torno al patrimonio se deben generar prácticas discursivas que hagan emerger formas de pensamiento contrahegemónicas, más sociales y comunitarias, menos individualistas y "cosificadas".

El problema, entonces, es icómo gestionar el patrimonio mientras tanto? ¿Esperando que se genere un aprecio social mayoritario que permita una gestión no coercitiva?, ¿o aplicando una política patrimonial orientada a que la gente se eduque y crezca socialmente en torno al patrimonio, a través de una construcción participativa del mismo? (Cuesta et al. 2009). El objetivo último no es la conservación del patrimonio, sino hacer que el aprecio hacia el patrimonio -incluso el criterio de poder decidir qué se quiere conservar y qué no, con todo lo que eso conlleva en cuanto a cambio de coordenadas culturales-, sea un discurso creado de forma participativa, no el resultado de una relación de dominio ${ }^{6}$. El patrimonio es un medio para la transformación social, y su aprecio será un beneficio más de dicha transformación. Incluso es factible que el propio concepto de patrimonio, algún día, deje de tener sentido, porque habrá una estima generalizada y una percepción global de "lo público", en el que estará incluido el patrimonio, sin ningún gran interés privado al que tenga que ser confrontado.

Mientras tanto, nos toca defender que el patrimonio es público, todo y siempre. ¿Cabe alguna duda de ello? Este aserto no es el alivio de las tensiones neoliberales que asaltan "lo público" en el Chile soñado por los Chicago boys, en la América Latina marcada por el Fondo Monetario Internacional (FMI) o, ahora mismo, en una Europa sacudida por la ortodoxia especulativa. En realidad esta dimensión pública es una consecuencia de todo lo anterior. Y tiene consecuencias en lo siguiente.

\section{$*$ Acciones}

Esta combinación de cualidades y características del patrimonio tiene implicaciones prácticas precisas para su gestión y para las disciplinas que intervienen en él.

\section{La práctica del patrimonio requiere transcender los límites disciplinares}

Requiere superar los marcos concretos de acción de cada disciplina. El patrimonio no entiende de arqueología, arquitectura, historia... Tampoco se puede clasificar con facilidad, a pesar del éxito y fuerza de las etiquetas consolidadas, en patrimonio arqueológico, construido, histórico, mueble, etnográfico, musical, u otras. Solo se puede hablar del patrimonio desde la interdisciplinariedad, algo que ya reclamaba Azkárate (2004) y que es frecuente argüir. Pero la interdisciplinariedad generalmente se entiende mal; es una noción a la que le falta apertura hermenéutica. Solo se puede ser interdisciplinar desde una interdisciplinariedad radical que disuelva los límites entre disciplinas y afronte los problemas empíricos, conceptuales, técnicos y metodológicos que el debate entre disciplinas genera.

\footnotetext{
${ }^{6}$ Lo que Smith (2011) denomina "Discurso Patrimonial Autorizado".
} 
Cualquier ejemplo consistente de práctica interdisciplinar permite reconocer que ésta es muy difícil, casi una quimera. No podemos minusvalorar el problema. Pero la solución está en otro sitio.

\section{Solo la transdisciplinariedad es una alternativa para trabajar y manejar el patrimonio}

Se requiere una práctica no orientada disciplinarmente, sino que apunte al problema que se pretende estudiar y gestionar. La cuestión no está en seguir siendo disciplinares, sino en marcar la agenda de trabajo desde la realidad y necesidades de cada objeto de estudio. La hegemonía dentro de un proyecto dado no debe venir marcada por las disciplinas que participan en él, y ni siquiera por su director y equipo (pues cada cual reproducirá entonces su disciplina), sino por el objeto o campo de trabajo. El plan de trabajo hay que diseñarlo desde el trabajo mismo, y no desde las disciplinas que participan en él.

\section{La construcción de un patrimonio público es un episodio concreto del reto mayor que es crear una ciencia pública}

Entendida en el doble sentido de "ciencia con la gente y ciencia realizada en la arena pública", generadora de un nuevo modelo de conocimiento que pueda favorecer y promover la innovación social7. La gestión integral del patrimonio forma parte de la pragmática de una ciencia pública porque es un escenario en el que se destilan, precipitan y posibilitan los cruces e interrelaciones entre racionalidades sin que dichas interrelaciones estén marcadas por el dominio de unas sobre otras. La confluencia de racionalidades, discursos, intenciones y expectativas que los diferentes individuos, grupos, sociedades y agentes se plantean en relación con el patrimonio, requiere articular diferentes razones e intereses, algo que solo se puede hacer estructurando los mecanismos de participación pública en el conocimiento, en su producción y en su uso, y no solo en su consumo y contemplación, tal y como proponen Criado-Boado y colaboradores (2010). Esto tiene también otra dimensión: los problemas prácticos de la gestión del patrimonio no son muy distintos (ni sus soluciones) de los de la ciencia en una sociedad avanzada. Existe una correspondencia entre ambos que,

7 Ver De Greiff y colaboradores (2011). sin embargo, no es reconocida ni por los gestores del patrimonio ni por los gestores de ciencia. La generalización en la gestión del patrimonio de fórmulas familiares y largamente ensayadas en la gobernanza de la ciencia (ya sean los "modernos" y lineales: concurrencia competitiva, organización público-privada, excelencia, evaluación externa de resultados, evaluación por pares, definición de indicadores; o los "posmodernos" y posnormales: community science, ciencia de garaje) aportarían una mejora sustantiva de aquella (Figura 5).

En este contexto, el patrimonio es un constituyente básico del nuevo tipo de conocimiento que necesitamos producir. Las sociedades del s. XXI precisan de una nueva forma de conocimiento $y$, no creemos que sea pomposo reconocerlo, a su construcción puede contribuir en gran medida, y por las razones esgrimidas aquí, el patrimonio y las prácticas relacionadas con éste. El patrimonio constituye un campo de experimentación esencial de los nuevos problemas y soluciones a los que se enfrenta una sociedad transmoderna, multicultural, globalizada, y sin embargo, sacudida por reclamaciones regionales, localistas, identitarias, que imponen con razón la necesidad de preservar y promover la diversidad cultural y la sostenibilidad social. Como acervo de tradiciones culturales dispares, en él confluyen y a veces conflictúan legitimidades diversas. Como encrucijada de racionalidades, requiere intertraducir todas las tradiciones existentes. Como valor simbólico, demanda la búsqueda de fórmulas de creación del sentido, consensuadas y negociadas, pero sin obviar que, en muchas ocasiones, esta encrucijada oculta la hegemonía de unas racionalidades sobre otras.

\section{Es necesario normalizar la gestión del patrimonio}

El patrimonio es conflictivo, como dijimos antes; debemos ser tolerantes y adoptar una posición positiva frente al hecho inevitable de que los valores patrimoniales vayan acompañados de cierta controversia o incluso conflictividad. Esto es asumible. Pero lo que no podemos soportar es que toda acción, intervención o proyecto patrimonial genere disputas y enfrentamientos, auténticas pulsiones suicidas que se saldan en contra de alguno de los intereses implicados $y$, habitualmente, del derecho básico del propio patrimonio que es su conservación, esto es, seguir existiendo-de-sí. Para ello es necesario normalizar el conflicto. Esto no quiere decir que las 


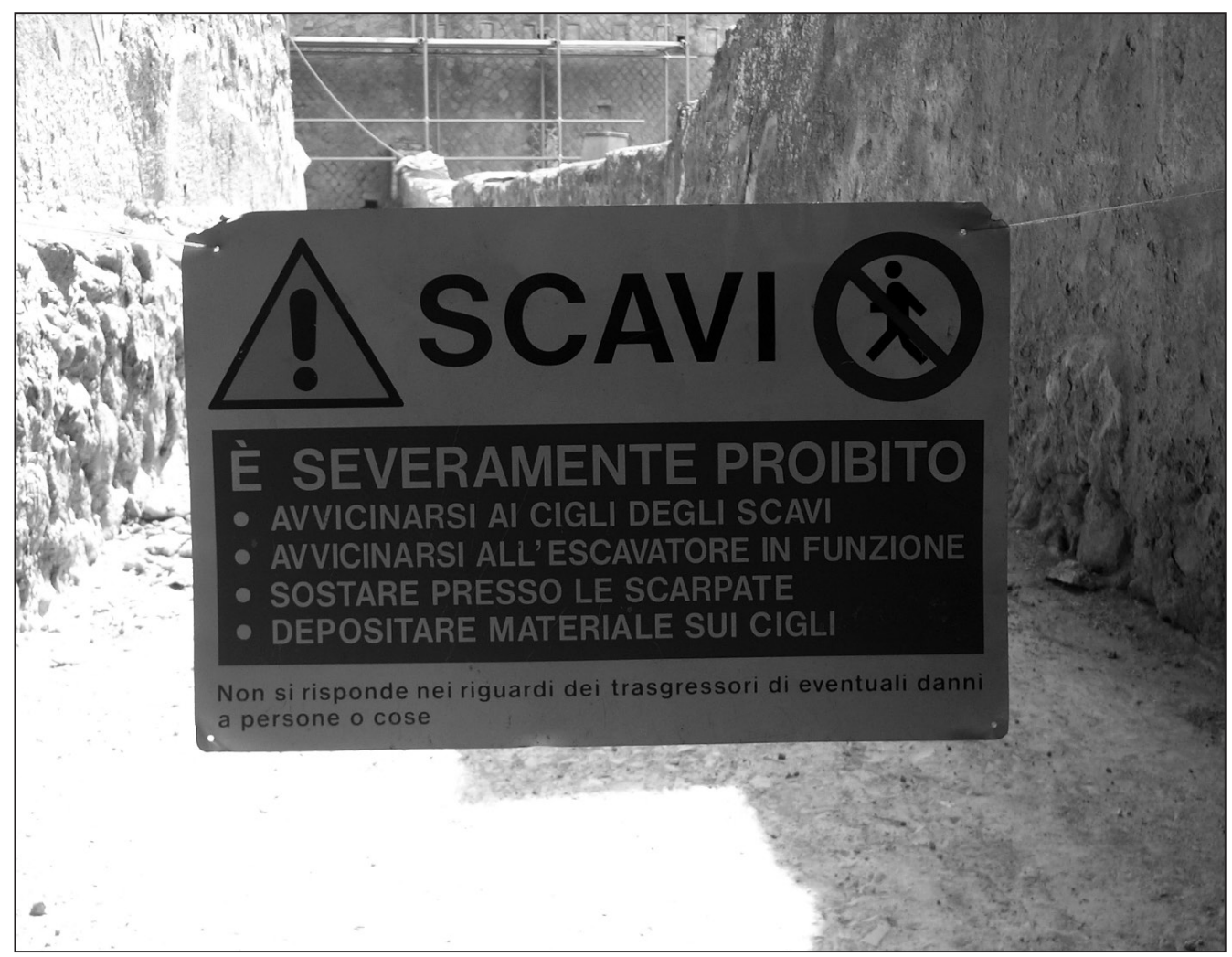

Figura 5. Así no. Típico cartel limitando el acceso al tajo en el que se desarrollan trabajos arqueológicos. Aunque este tipo de precauciones eran y son aún muy normales, ha llegado el momento en el que (por imperativo teórico, práctico y ético) no se pueden sostener estructuras de cierre de la experiencia patrimonial y arqueológica.

fracturas y contradicciones deban ser ocultadas bajo una práctica discursiva uniformizadora, hegemónica, sino que tenemos la obligación de buscar fórmulas multivocales y participativas de producción, uso y gestión del patrimonio compatibles con las necesidades de conservación, más técnicas y asépticas y, si se quiere, más acordes con un discurso objetivista e instrumentalizante.

\section{Se debe implantar el principio de corresponsabili- dad en el estudio y gestión del patrimonio}

Es necesaria una corresponsabilidad que propicie la interacción entre administraciones: la local, la regional, la estatal, la supraestatal ${ }^{8}$, entre éstas y los colectivos pro-

\footnotetext{
8 No olvidemos, por ejemplo, que la protección y gestión del rico patrimonio subacuático mundial es una problemática que no se puede resolver en la escala de los gobiernos nacionales y que desafía los límites y posibilidades de la gobernanza planetaria, como
}

fesionales, entre éstos y los agentes públicos y comunitarios. En particular, la necesaria coordinación entre departamentos (ministerios, concejalías, entre otros) que se relacionan con el patrimonio, es una tarea pendiente: la administración del patrimonio no se puede distanciar de otras políticas sectoriales como medio ambiente, suelo, urbanismo, vivienda; tampoco ciencia, educación y cultura.

\section{El valor del patrimonio implica su valor económico}

Es algo que hoy no podemos dejar de reconocer. No sirven posiciones presuntamente asépticas y virginales, que aún siguen preponderando en muchos ambientes, sobre

Odissey se empeña en demostrarnos sucesivamente, a pesar de que el fallo de los tribunales norteamericanos en su contra y en favor de la reclamación del Estado español en el caso de la fragata Las Mercedes les haya momentáneamente cortado las alas. 


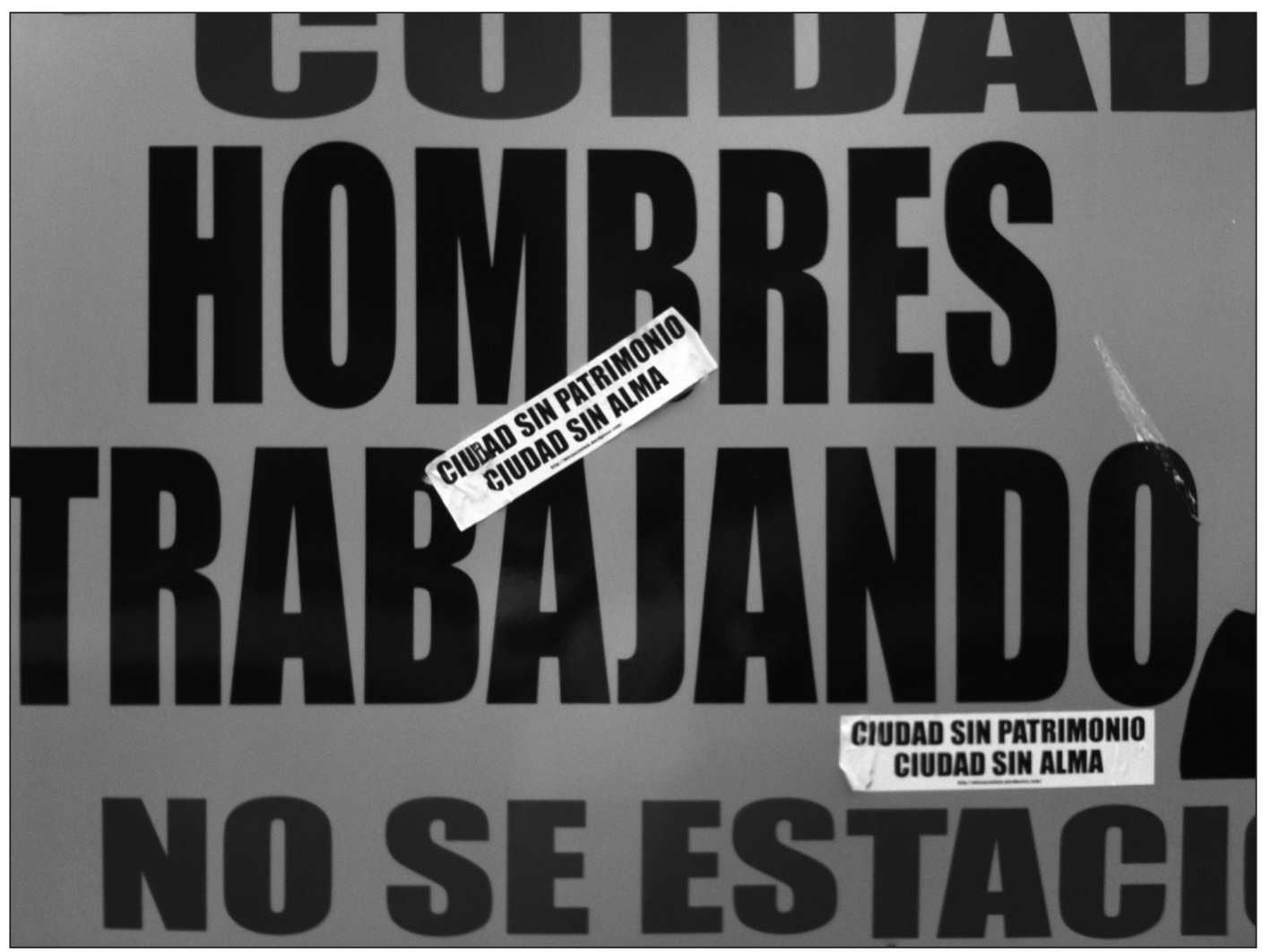

Figura 6. Salvo que queramos tomarnos totalmente en serio la letra del cartel para alejarnos de prisa de todas las prácticas que aún son sexistas y reifican una realidad machista, esto es lo que no queremos: que el público se tenga que alejar de allí donde trabajamos. Fotografía tomada en una valla que cerraba una obra de rehabilitación en el casco histórico de Ciudad de Panamá. No es coincidencia que el cartel esté sobremontado por pegatinas reclamando el valor y uso del patrimonio.

todo académicos. El tema no está exento de riesgos: qué valor, cómo se establece, en beneficio de quién, con qué modelo de gestión se rentabiliza, qué plan de negocio es asumible, cómo sustraemos los bienes patrimoniales del proceso de "cosificación" y comercialización generalizado, dónde situamos la frontera entre esta dimensión del patrimonio y la tendencia ilimitada a la especulación. Sin obviar estos problemas, sino enfrentándonos, hay que reconocer y estudiar los procesos de producción de valor económico relacionados con el patrimonio. Y esto sitúa como una prioridad la necesidad de desarrollar una economía del patrimonio y una sociología del mercado de trabajo, de la economía política relacionada con el patrimonio. Es algo aún pendiente, una necesidad infradesarrollada, aunque existan intentos de sistematización como los de Vicente (2007). Las confluencias de esta línea de investigación con la economía de la cultura, del turismo e, incluso, del medioambiente, son precisas y reales, por lo que la creación de una economía del patrimonio podría avanzar en gran medida basando sus modelos de referencia en esos otros campos (auténticas subdisciplinas de la teoría económica) que ya están relativamente consolidados. Una economía del patrimonio orientada a entender los procesos de producción de valor en torno a él puede contribuir a la generación de nuevos vectores económicos, alternativos a un sistema de libre mercado cortoplacista. La economía es una dimensión básica de la condición humana, un factor ineludible del ser y estar en el mundo; el mercado capitalista, no. Es un producto histórico que puede ser transformado. Por lo tanto, pensar en la dimensión económica del patrimonio es también pensar en nuevos modelos económicos para la sociedad, contribuyendo así a la reorientación de esos modelos de referencia más avanzados (Figura 6). 


\section{* Prioridades}

Arriesgándonos a ser parciales, si tuviéramos que derivar de los comentarios anteriores una lista de temas y aspectos que sea necesario considerar con urgencia dentro del estudio del patrimonio y del mejoramiento de sus fórmulas de gestión, seleccionaríamos los siguientes aspectos porque constituyen temáticas que no han sido tratadas en detalle o están aún pendientes:

- Perfeccionamiento de los sistemas de registro, documentación e inventario, incluyendo el manejo de información visual y oral.

- Identificación, caracterización y protección de los paisajes culturales, aunque en realidad el tema es más amplio, pues la auténtica urgencia es comprender la territorialización del patrimonio y sus dimensiones espaciales, que son una prolongación de sus circunstancias materiales.

- Análisis de la correlación del patrimonio con los procesos de constitución de la memoria, las identidades y los valores. Esto involucra tanto el examen crítico de los esencialismos hegemónicos vinculados al patrimonio, como el descubrimiento de los procesos de negociación entre tradiciones culturales.

- Análisis de procesos de patrimonialización, es decir, cómo se constituye el patrimonio y, en particular, cómo surgen nuevos patrimonios en la actualidad y cuál es el destino de los patrimonios indeseados de vieja y nueva recreación.

- Estudio y gestión del patrimonio inmaterial, o lo que también se denomina etnológico, intangible, simbólico, incluyendo en particular su recuperación y sin dejar de reconocer que todo elemento patrimonial tiene siempre, una dimensión inmaterial.
- Reconocimiento y análisis de los procesos de participación social y de co-construcción del patrimonio y de los modelos o alternativas de gestión y socialización del mismo.

- Posibilidades y límites de la producción de múltiples narrativas en relación con el patrimonio.?

- Prospectiva de las medidas necesarias y posibles para estimular la innovación social, incluyendo los proyectos emprendedores y el surgimiento de nuevos valores económicos en relación con el patrimonio.

\section{$*$ Consecuencias}

Todo lo anterior se resume en una simple idea, o mejor, ideal. El futuro del patrimonio está entreverado con el necesario proceso de resocialización de las prácticas y las políticas que la sociedad necesita en la actualidad. El desafío, entonces, es doble: en primer lugar, estriba en cómo propiciar la consolidación de la dimensión pública de los bienes patrimoniales en un contexto marcado por el reflujo de los valores públicos y por la gestión de una crisis que va camino de amortizarse en beneficio de los poderes que la desencadenaron; $y$, en segundo lugar, en cómo convertir al campo patrimonial en un activo campo de agencia social alternativa y contra-hegemónica.

El enunciado que da título a este texto no solo se refiere a la noción que hemos defendido aquí de que lo que ahora es patrimonio antes fue otra cosa distinta; sino que también quiere remarcar que, posiblemente, en un futuro utópico y necesario, la gestión patrimonial, y el propio patrimonio, sean algo esencialmente distintos a lo que hasta aquí hemos estado haciendo.

9 Un buen ejemplo de ello es el proyecto y vídeo Los Narradores del Caraguatá, accesible en el sitio web: http://hdl.handle. net/10261/25625. 


\section{* Referencias citadas}

ALBA RICO, S., 2012. El paisaje: La mirada como derrota. http://www. rebelion.org/noticia.php?id=160639

AZKÁRATE, A., 2004. La interdisciplinariedad cuna concesión al lenguaje políticamente correcto? En Segunda Bienal de la Restauración Monumental. Qué está pasando. Condicionantes, teoría y praxis actuales del ejercicio de la restauración monumental, J. I. Lasagabáster-Gómez (Dir.), pp. 41-44. Fundación Catedral Santa María, Vitoria-Gasteiz.

BARREIRO, D., 2003. Arqueología y pragmatismo crítico. Hacia la renovación axiológica de la arqueología. Claves de Razón Práctica 133: 36-41. http://hdl.handle.net/10261/16054

2012. Arqueología aplicada y patrimonio: Memoria y utopía. Complutum 23 (2): 29-46.

BERMAN, M., 1988. Todo lo sólido se desvanece en el aire. La experiencia de la modernidad. Siglo XXI, Madrid.

CHESWORTH, W., 2012. Living on an overloaded world: The long view of the Neolithic revolution. En Petroglifos, paleoambiente $y$ paisaje, F. Criado-Boado, A. Martínez-Cortizas y M. GarcíaQuintela (Eds.), Tapa 42: 293-301. Consejo Superior de Investigaciones Científicas, Madrid.

CRIADO-BOADO, F., 1996. El futuro de la arqueología ¿la arqueología del futuro? Trabajos de Prehistoria 53 (1): 15-35. http://hdl. handle.net/10261/12248

CRIADO-BOADO, F., D. BARREIRO, X. L. ARMADA, R. BLANCO-ROTEA, M. COSTA-CASAIS, C. GONZÁLEZ-PÉREZ, C. PARCERO-OUBIÑA, M. P. PRIETO-MARTÍNEZ y C. SÁNCHEZ-CARRETERO, 2008. The Heritage Laboratory Strategic Plan:An interdisciplinary research line on cultural heritage. http://hdl. handle.net/10261/12211

CRIADO-BOADO, F., A. CORSÍN, A. DE GREIFF, C. RODRÍGUEZ, E. MEDINA, J. TAGUEÑA, J. MARTÍN, L. VELHO, L. OOSTERBEEK y F. ORTEGA, 2010. Definición del Área de Ciencia y Sociedad. Documento aprobado en la Asamblea General de CYTED celebrada en Urubamba, Perú, el 27 de noviembre de 2009. CYTED, Programa Iberoamericano de Ciencia y Tecnología para el Desarrollo. http://hdl.handle. net/10261/20961

CUESTA, A. V., J. DiMURO, C. GIANOTI y M. MUTTONI, 2009. De la investigación a la construcción participativa del patrimonio. Un programa de educación patrimonial y divulgación de la cultura científica en Uruguay. Arkeos 4 (11). http://mileto. pucp.edu.pe/arkeos/content/view/225/26/
DE GREIFF, A. y O. J. MALDONADO, 2011. "Apropiación fuerte" del conocimiento: Una propuesta para construir políticas inclusivas de ciencia, tecnología e innovación en América Latina. En Estudio social de la ciencia y la tecnología desde América Latina, A. Arellano y P. Kreimer (Eds.), pp. 209-262. Siglo del Hombre Editores, Bogotá.

GIANOTTI, C. y MARTÍN DABEZÍES (Eds.), 2011. Huellas de la memoria.Anaina 1.http://hdl.handle.net/10261/41123

GONZÁLEZ-MÉNDEZ, M., 1999. Investigación y puesta en valor del patrimonio histórico: Planteamientos y propuestas desde la arqueología del paisaje. Tesis doctoral. Historia I de la Facultade de Xeografía e Historia de la Universidad de Santiago de Compostela, Santiago de Compostela. http://hdl.handle. net/10261/28279

GONZÁLEZ-PÉREZ, C. y C. PARCERO-OUBIÑA, 2011. A conceptual model for cultural heritage. Definition and motivation. Revive the past. Proceedings of the 39 th Conference in Computer Applications and Quantitative Methods in Archaeology, Beijing.

GONZÁLEZ-RUIBAL, A., 2009. Topography of terror or cultural heritage? The monuments of Franco's Spain. En Europe's deadly century. Perspectives on 2oth century conflict heritage, N. Forbes, R. Page y G. Pérez (Eds.), pp. 65-72. English Heritage, Kemble Drive, Swindon. http://hdl.handle.net/10261/21335

HARVEY, D., 2001. Heritage pasts and heritage presents: Temporality, meaning and the scope of heritage studies. International Journal of Heritage Studies 7 (4): 319-338.

HERNÁNDEZ, G. M., 2008. Un zombi de la modernidad: El patrimonio cultural y sus límites. La Torre del Virrey 5: 27-38.

KAAL, J., Y. CARRIÓN-MARCO, E. ASOUTI, M. MARTÍNSEIJO, A. MARTÍNEZ-CORTIZAS, M. COSTA-CASAIS y F. CRIADO-BOADO, 2011. Long-term deforestation in NW Spain: Linking the Holocene fire history to vegetation change and human activities. Quaternary Science Review 30 (1-2): 161-175.

SÁNCHEZ-CARRETERO, C., 2012. Hacia una antropología del conflicto aplicada al patrimonio. En Geopolíticas patrimoniales: De culturas, naturalezas e inmaterialidades. Una mirada etnográfica, B. Santamarina (Ed.), pp. 195-210. Editorial Germania, Colección Antropológicas, Valencia.

SANTAMARINA, B., 2005. Una aproximación al patrimonio cultural. En La memoria construida: Patrimonio cultural y modernidad, G. M. Hernández (Coord.), pp. 21-51. Tirant lo Blanch, Valencia. 
SMITH, L., 2006. Uses of heritage. Routledge, Londres.

2011. El "espejo patrimonial”. ¿Ilusión narcisista o reflexiones múltiples? Antípoda Revista de Antropología y Arqueología 12:39-63.

SOLLI, B., M. BURSTRÖM, E. DOMANSKA, M. EDGEWORTH, A. GONZÁLEZ-RUiBAL, C. HOLTORF, G. LUCAS, T. OESTIGAARD, L. SMITH y C. WITMORE, 2011. Somereflections on heritage and archaeology in the Anthropocene. Norwegian Archaeological Review 44 (1): 40-88.
TUNBRIDGE, J. E. y G. J. ASHWORTH, 1996. Dissonant heritage: The management of the past as a resource in conflict. John Wiley and Sons Ltd., Chichester.

VICENTE, E., 2007. Economía del patrimonio y politicas patrimoniales: Un estudio de la política del patrimonio histórico en Castilla y León. Instituto de Estudios Fiscales, Madrid.

WATERTON, E.y L. SMITH, 2009. There is no such thing as heritage. En Taking archaeology out of heritage, E. Waterton y L. Smith (Eds.), pp. 10-27. Cambridge Scholars Publishing, Newcastle upon Tyne. 